\title{
Herzlich willkommen in Essen, einem der Top-Medizinstandorte Deutschlands und dem führenden Gesundheitsstandort im Ruhrgebiet!
}

Wir freuen uns darauf, Sie hier vom 30. September bis zum 3. Oktober 2020 zur 57. Jahrestagung der Gesellschaft für Pädiatrische Radiologie (GPR) zu begrüßen! Premiumpartner ist das Universitätsklinikum Essen, das anstrebt, zukünftig mit einem eigenen Institut eine Vorreiterrolle bei der durch künstliche Intelligenz gestützten medizinischen Versorgung zu übernehmen mit dem Ziel, ein starker Dienstleister onkologischer Super-Diagnostics zu werden.

\section{Zukunft Kinderradiologie}

Unter der Leitidee „ZUKUNFT KINDERRADIOLOGIE“ laden wir Sie ein, sich über die Bedeutung künstlicher Intelligenz in der Medizin und den aktuellen Forschungsstand zu informieren, einen Blick in die Welt von morgen zu werfen und die Poten- ziale der digitalen Revolution mit Fokus auf die Kinderradiologie kennenzulernen.

Sicher ist: KI wird auch in der Pädiatrischen Radiologie eine immer wichtigere Rolle spielen. Doch viele Fragen sind noch offen: Wie lässt sich mithilfe dieser Technologie die Diagnostik verbessern? Welche Probleme müssen - insbesondere in der Kinderradiologie - noch gelöst werden?

\section{Zukunft Gastrointestinale Bildgebung und Neuroradiologie}

Weitere wichtige Zukunftsthemen des Kongresses sind die gastrointestinale Bildgebung und die Neuroradiologie bei Kindern. Im Mittelpunkt des Kongresses stehen die technischen Weiterentwicklun- gen und die Erweiterung des Einsatzspektrums der Magnetresonanztomografie. Moderne MRT-Systeme lassen mit ihren schnellen Sequenzen heute oftmals sogar eine narkosefreie Diagnostik bei Kindern zu. Eine wesentlich verbesserte Detailtiefe bei den Darstellungsmöglichkeiten von Geweben ermöglicht klinischen Kolleginnen und Kollegen neue Ansätze der Behandlung. Was bedeutet das für die Zukunft der Pädiatrie?

\section{Das Programm}

Das umfassende Kongressprogramm mit Ärzte-/MTRA-Fortbildungen und dem wissenschaftlichen Kongress ist so konzipiert, dass Veranstaltungselemente ideal miteinander kombiniert werden können und niemand etwas verpasst. 
Mit herzlichen Grüßen aus dem Ruhrgebiet Glückauf!

Ihr Bernd Schweiger

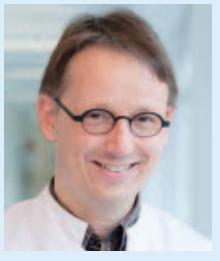

Dr. med. Bernd Schweiger

Kongresspräsident 2020

\section{Call-for-Abstract 57. Jahrestagung in Essen}

Ab sofort ist die Abstract-Einreichung für die GPR-Jahrestagung geöffnet. Bis zum 20. Mai 2020 können Sie Ihre wissenschaftlichen Abstracts und Kasuistiken einreichen. Weitere Informationen zu den Themen und die Einreichung finden Sie hier:

https://gpr-jahrestagung.de/

\section{Science-Slam Kinderradiologie}

Erstmalig veranstaltet die GPR auf ihrer Jahrestagung einen Science-Slam Kinderradiologie. Weitere Informationen zur Veranstaltung und zur Teilnahme als Slammer finden Sie hier https://gpr-jahrestagung.de/

\section{SAVE THE DATE}

57 Jahrestagung der Gesellschaft

5 - für Pädiatrische Radiologie

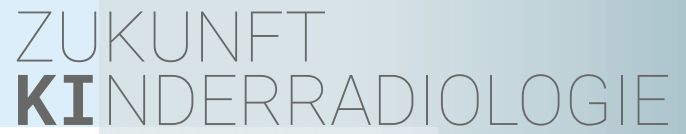

\subsection{9. - 03.10.2020, Essen}

Kongresspräsident Dr. Bernd Schweiger

- Künstliche Intelligenz

- Gastro-intestinale Bildgebung

- Neuroradiologie

NEU: Kinderradiologischer Science-Slam!

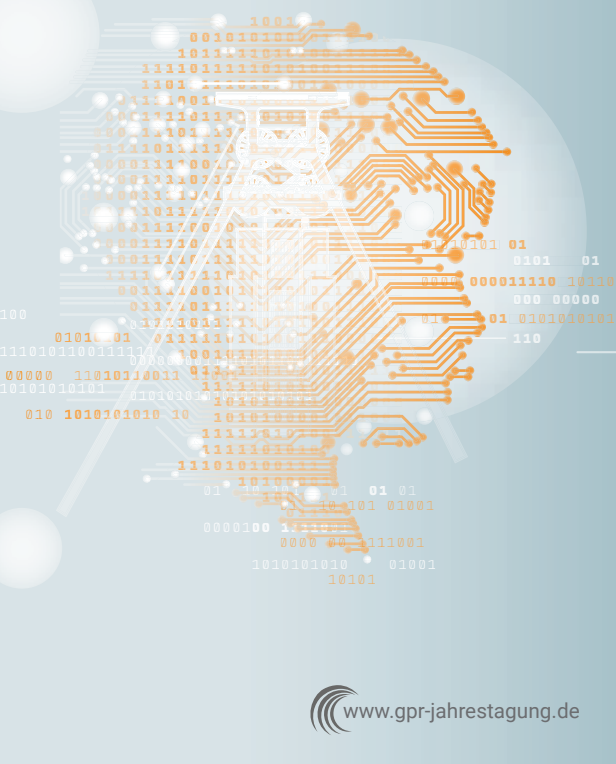

\section{SAVE THE DATE}

57 Jahrestagung der Gesellschaft

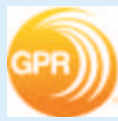

51. für Pädiatrische Radiologie

Liebe Kolleginnen und Kollegen,

zur 57. Jahrestagung der Gesellschaft für

Pädiatrische Radiologie möchte ich Sie

vom 30.09. bis zum 03.10.2020 ganz herzlich nach Essen einladen.

\section{September 2020}

Fortbildungstag für Ärztinnen und Ärzte

01. - 03. Oktober 2020

Wissenschaftlicher Kongress

Schwerpunkte:

- Künstliche Intelligenz

- Gastro-intestinale Bildgebung

- Neuroradiologie

\section{Oktober 2020}

MTRA-Fortbildung

Ich hoffe, Sie im nächsten Jahr bei uns

im Ruhrgebiet begrüßen zu können

und verbleibe mit einem herzlichen Glückauf!

Strat flewig

Dr. Bernd Schweiger

Kongresspräsident - Universitätsklinikum Essen

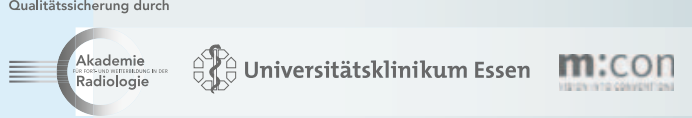

